EPiC Series in Engineering
Volume 3, 2018, Pages 1713-1717
HIC 2018. 13th International
Conference on Hydroinformatics

\title{
Pressure Sensors Positioning for Leakages Detection under Uncertain Demands
}

\author{
Valeria Puleo $^{1 *}$, Gabriele Freni ${ }^{2}$ and Goffredo La Loggia ${ }^{1}$ \\ ${ }^{1}$ Department of Civil, Environmental, Aeronautic, and Chemical Engineering, University of \\ Palermo, Viale delle Scienze, Ed. 8, Palermo, Italy \\ ${ }^{2}$ School of Engineering and Architecture, University of Enna "Kore", Cittadella Universitaria, \\ Enna, Italy \\ valeria.puleo@unipa.it, gabriele.freni@unikore.it, \\ goffredo.laloggia@unipa.it
}

\begin{abstract}
The pressure sensors positioning is a crucial step for leakages detection. The optimal positioning of monitoring sensors, or simply sampling design, has been previously addressed with respect to several purposes. The proposed methodology aims to select the pressure monitoring nodes for leakages detection by coupling the water distribution network hydraulic simulation model with the identifiability analysis. The nodes selection is done among those which are more sensitive with respect to different leakages positions and uncorrelated from each other to avoid redundant information. The parameter uncertainty effect on the results is also investigated. The method is applied to the benchmark network Apulian.
\end{abstract}

\section{Introduction}

To reduce leakages several methods and practices are available, and several efforts have been done to increase the knowledge by means of experimental (De Marchis, Fontanazza, Freni, Notaro, \& Puleo, 2016) and numerical modelling (Cassa, van Zyl, \& Laubscher, 2010).

Model-based techniques, including WDNs hydraulic modelling coupled with several algorithms for leakages detection, can allow more effective and less costly water losses control strategies.

The optimal positioning of monitoring sensors, or simply sampling design, has been previously addressed with respect to several purposes (Bush \& Uber, 1998) such as monitoring for baseline system characteristics, detection of contamination events or leaks, compliance for maintenance of system performance and calibration of network models. Several sampling design techniques have been developed for model calibration (Bush \& Uber, 1998; de Schaetzen, Walters, \& Savic, 2000), for

${ }^{*}$ Corresponding author 
contamination events (Ostfeld \& Salomons, 2004) and leakages detections (Farley, Mounce, \& Boxall, 2010).

In Steffelbauer, Neumayer, Günther, \& Fuchs-Hanusch (2014) the sensor placement was solved through a non-binarized leak sensitivity matrix with a projection-based leak isolation approach. Moreover, the hydraulic model parameters uncertainty effects on the measurements have been considered in the analysis. A differential evolution algorithm performed the leakage localization. The results showed that the most sensitive nodes to the leakages can also be to the demand fluctuations. Blesa, Nejjari, \& Sarrate (2014) defined a robustness percentage index to evaluate the sensor placement strategies based on the fault sensitivity matrix for different leak magnitudes and operating scenarios (e.g. changes in demands). A clustering analysis reduced the number of the candidate sensors. A significative variation on the leak localization was observed when the operating points changed.

In the present paper the identifiability analysis is applied to select the most reliable nodes for pressure sensor positioning under uncertain demands.

\section{Methods}

The identifiability analysis is applied to evaluate the nodes where the pressures (output variables) are more sensible to leakages (model input parameters) variation, to arrange pressure sensors for the leak detection. A water distribution network hydraulic model can be described by a general set of equations $y=f(\vartheta)$ where $y$ is the vector of the $n$ output variables and $\vartheta$ the vector of the $m$ independent model parameters. Sensitivity functions can be defined to have information about the raw dependency of the output with model parameters (eq. 1):

$$
s_{i j}=\frac{\Delta \vartheta_{j}}{y s_{i}} \frac{\delta y_{i}}{\delta \vartheta_{j}}
$$

where $\Delta \vartheta_{j}$ is the variation range of the $j^{\text {th }}$ parameter; $y s_{i}$ is the reference value of the output variable $y_{i}$. The sensitivity functions can be allocated in the $S$ matrix: each entry in this matrix considers the variations of pressures between the no-leak scenario and the leak-scenarios.

The sensitivity functions are used to evaluate the identifiability indices providing the number and the list of the pressure monitoring nodes for the specific water distribution network model. The analysis starts with a sensitivity ranking of the pressure monitoring nodes by averaging the sensitivities of the modelling output $y_{i}$ to the variations of the $m$ parameters (eq. 2).

$$
\bar{s}_{i}=\frac{1}{m} \sum_{j=1}^{m}\left|s_{i j}\right|
$$

Starting from this ranking is possible to identify a subset of nodes by applying a threshold which should be carefully chosen to avoid deleting too many or too few nodes. Among this subset, several combination of nodes are investigated using the identifiability criteria based on the analysis of the Fisher Information Matrix (FIM) which can be written as follows:

$$
F I M=\left[S \cdot S^{T}\right]
$$

Starting from the FIM, the identifiability index (eq. 4) is formulated as the combination of the normalised determinant $(\operatorname{norm} D)$ and condition number $(\bmod E)$ criteria (Freni, Mannina, \& Viviani, 2011). The nodes combination providing the highest values of the identifiability index is chosen as monitoring subset. 


$$
D E=\frac{n o r m D}{\bmod E}=\frac{\max \left(D \cdot\|\vartheta\|^{2}\right)}{\min \sqrt{\frac{\max (E V[F I M])}{\min (E V[F I M])}}}
$$

where $\|\vartheta\|^{2}$ is the Euclidean norm of the vector of the parameters estimated at the mean point of the parameter-variation range; $\max (E V[F I M])$ and $\min (E V[F I M])$ are the maximum and minimum eigenvalues (EV) of the FIM, respectively.

To evaluate the effect of uncertain input parameters on the model outputs an uncertainty analysis is performed through the Monte Carlo Simulations (MCS) converging to the exact uncertainty estimates if many parameter sets are simulated. Namely, the effect of uncertain demands on the pressures as well as on the identifiability results is analysed. The demand is randomly drawn from a normal distribution with a mean $\mu$ and a standard deviation $\sigma$. Subsequently, EPANET is called using these randomly distributed demands to perform a hydraulic simulation for obtaining the resulting pressures $\mathrm{p}_{\mathrm{j}}$ at every node $\mathrm{j}$ in the system. This procedure is repeated mcsteps-times to get the approximate probability distributions $\mathrm{P}\left(\mathrm{p}_{\mathrm{j}}\right)$ of $\mathrm{p}_{\mathrm{j}}$ for every possible measurement position in the system. Once $\mathrm{P}\left(\mathrm{p}_{\mathrm{j}}\right)$ is calculated, the standard deviation $\sigma_{\mathrm{j}}$ is taken from this distribution serving as penalty coefficient of the pressures in point $\mathrm{j}$. The higher $\sigma_{\mathrm{j}}$, the noisier the signal is at this point, the less ideal this location is for pressure measurements.

\subsection{The Apulian network}

The Apulian network is a small-sized network constituted of 23 nodes and 34 pipes. It is fed by a reservoir with fixed hydraulic head, there are no tanks, valves or pumping stations. The leakages are modeled as new nodes, placed halfway in the pipes except for the pipe connected to the reservoir, by using the EPANET emitter function. The leak-nodes elevations are computed as average of the elevations of the pipe connecting nodes. Therefore, the simulated leakage-nodes are 33 . The network scheme is depicted in Figure 1.

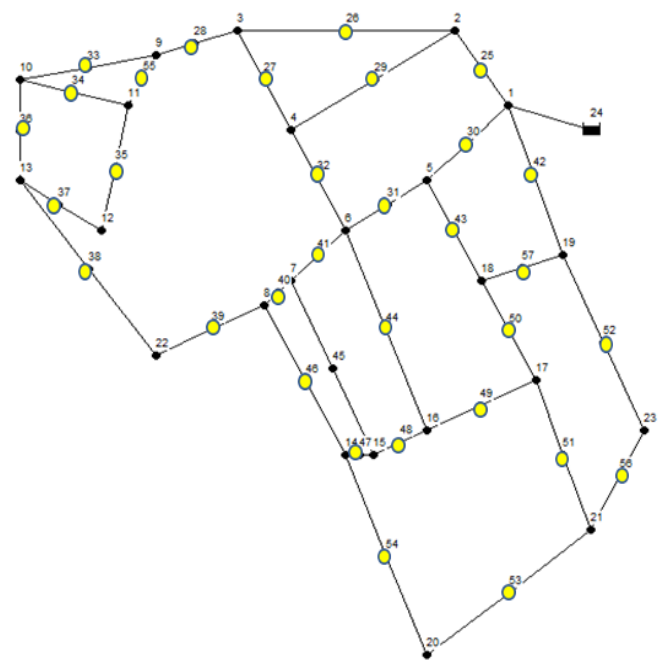

Figure 1: The Apulian network with emitter nodes (in yellow) 


\section{Results}

The eligible pressure monitoring nodes are firstly compared according to the value of the mean of the average sensitivity index, $\bar{s}_{i}$. The results are reported in Table 1 with regards to Test 1 (certain demands), Test 2 (uncertain demands; without penalty) and Test 3 (uncertain demands; with penalty). Only the first five candidate nodes are shown with regard to the index $\overline{\boldsymbol{s}}_{\boldsymbol{i}}$. Test 1 and 2 show the same nodes ranking; as expected, the most sensitive nodes to the leakages are also to the demand variations. According to the methodology, the penalty coefficient is calculated, and the resulting nodes selection is reported in the Test 3 . The node 23, which is the best position for pressure sensor for Test 1 and 2 , is the worst for Test 3; while the node 12 gains the first position.

Starting from these results the identifiability index $D E$ is applied to have the final combination of the nodes for a fixed number of installed sensors. In particular, several subset have been analysed. The resulting sensors placement is reported in Table 2. The same subset of nodes was identified for the simulated uncertainty level also when the penalty coefficients are considered. Nodes 13 and 15 are excluded while node 4 is chosen although its sensitivity index is lower. The identifiability index identifies the subset of eligible points which are more sensitive to the leakages and also provide less correlated measurements.

Table 1: The average sensitivity index $\overline{\boldsymbol{s}}_{\boldsymbol{i}}$ for Test 1 (certain demands), Test 2 (uncertain demands; without penalty) and Test 3 (uncertain demands; with penalty)

\begin{tabular}{ccccccc}
\hline \multirow{2}{*}{ Ranking } & Test 1 & \multicolumn{3}{c}{ Test 2} \\
\cline { 2 - 7 } & node ID & $\overline{\boldsymbol{s}}_{\boldsymbol{i}}$ & node ID & $\overline{\boldsymbol{s}}_{\boldsymbol{i}}$ & node ID & $\overline{\boldsymbol{s}}_{\boldsymbol{i}}$ \\
\hline 1 & 23 & 1.424 & 23 & 1.595 & 12 & 1.353 \\
2 & 12 & 1.410 & 12 & 1.546 & 16 & 1.305 \\
3 & 13 & 1.309 & 13 & 1.369 & 13 & 1.248 \\
4 & 16 & 1.298 & 16 & 1.304 & 15 & 1.235 \\
5 & 21 & 1.277 & 21 & 1.293 & 23 & 1.234 \\
\hline
\end{tabular}

Table 2: The sensors placement using the identifiability index for Test 1 (certain demands), Test 2 (uncertain demands; without penalty) and Test 3 (uncertain demands; with penalty)

\begin{tabular}{clll}
\hline $\begin{array}{l}\mathrm{n}^{\circ} \text { of } \\
\text { installed } \\
\text { sensors }\end{array}$ & Test 1 & \multicolumn{1}{c}{ Nodes ID } \\
\cline { 2 - 4 } & 12,23 & Test 2 & Test 3 \\
\hline 2 & $4,12,23$ & 4,23 & 12,23 \\
3 & $4,12,16,23$ & $4,12,16,23$ & $4,12,23$ \\
4 & $4,12,16,20,23$ & $4,12,16,20,23$ & $4,12,16,23$ \\
5 & & $4,12,16,20,23$ \\
\hline
\end{tabular}

\section{Conclusions}

The identifiability analysis was investigated as sampling design method for the pressure monitoring sensor positioning. The main objective of the analysis was the selection of the sensor location for leakages detection. The uncertainty analysis allowed to investigate the effect of the 
uncertainty linked to demand on the positioning methods. The sensitivity functions changed with the uncertainty level tested, whereas FIM results did not show such dependency.

Further development in research will be done with regard to the uncertainty analysis and the detection of leakages for fixed sensor positioning.

\section{Reference}

Blesa, J., Nejjari, F., \& Sarrate, R. (2014). Robustness Analysis of Sensor Placement for Leak Detection and Location under Uncertain Operating Conditions. Procedia Engineering, 89, $1553-1560$.

Bush, C. A., \& Uber, J. G. (1998). Sampling design methods for water distribution model calibration. Journal of Water Resources Planning and Management-Asce, 124(6), 334-344.

Cassa, A. M., van Zyl, J. E., \& Laubscher, R. F. (2010). A numerical investigation into the effect of pressure on holes and cracks in water supply pipes. Urban Water Journal, 7(2), 109-120.

De Marchis, M., Fontanazza, C. M., Freni, G., Notaro, V., \& Puleo, V. (2016). Experimental Evidence of Leaks in Elastic Pipes. Water Resources Management, 30(6), 2005-2019.

de Schaetzen, W. B. F., Walters, G. A., \& Savic, D. A. (2000). Optimal sampling design for model calibration using shortest path, genetic and entropy algorithms. Urban Water, 2(2), 141-152.

Farley, B., Mounce, S. R., \& Boxall, J. B. (2010). Field testing of an optimal sensor placement methodology for event detection in an urban water distribution network. Urban Water Journal, 7(6), 345-356.

Freni, G., Mannina, G., \& Viviani, G. (2011). Assessment of the integrated urban water quality model complexity through identifiability analysis. Water Research, 45(1), 37-50.

Ostfeld, A., \& Salomons, E. (2004). Optimal layout of early warning detection stations for water distribution systems security. Journal of Water Resources Planning and Management-Asce, 130(5), 377-385.

Steffelbauer, D., Neumayer, M., Günther, M., \& Fuchs-Hanusch, D. (2014). Sensor Placement and Leakage Localization Considering Demand Uncertainties. Procedia Engineering, 89, 11601167.Blesa, J., Nejjari, F., \& Sarrate, R. (2014). Robustness Analysis of Sensor Placement for Leak Detection and Location under Uncertain Operating Conditions. Procedia Engineering, 89, 1553-1560. 